\title{
Measles: an important cause of fever and rash in a returned traveller
}

G Khai Lin Huang MBBS(Hons), BMedSci Infectious Diseases Registrar ${ }^{1}$

Katherine A Bond BSci, MBBS(Hons), DTM\&H Infectious Diseases Registrar, and Senior Public Health Officer $^{2}$

Nicholas H Hewitt

MBBS(Hons), FRACP Infectious Diseases Registrar ${ }^{1}$

Paul DR Johnson MBBS, PhD, FRACP Deputy Director, and Professor $^{3}$

1 Infectious Diseases Department, Austin Health, Melbourne, VIC.

2 Communicable Diseases Prevention and Control, Victorian Department of Health, Melbourne, VIC.

3 University of Melbourne, Melbourne, VIC.

khai.huang@gmail.com

doi: 10.5694/mjal4.00669

\section{Clinical record}

A previously well 21-year-old woman went to her general practitioner in September 2013, before a holiday to Bali, Indonesia, and was vaccinated for hepatitis A and typhoid. Malaria prophylaxis was not prescribed, as her intended destinations were deemed to confer a low risk of acquiring malaria. Notably, she had not received routine childhood vaccinations because of parental preference, receiving only "homeopathic immunisation" in infancy.

During October 2013, she spent 11 days in Bali, primarily in large holiday resorts in the Kuta district, but she did travel to a rural village. She reported receiving several mosquito bites during her trip.

Two days before her return to Australia, she developed fever, vomiting and diarrhoea. These symptoms continued until she presented to her GP 1 day after returning to Australia. She was prescribed metronidazole and loperamide and her diarrhoea resolved, but nausea and lack of appetite persisted. The following day she developed chills with associated headache, myalgias and cough - these symptoms continued until she presented to hospital 7 days after her return to Australia.

On initial review in hospital, 9 days after the onset of her illness, the patient was febrile $\left(38.5^{\circ} \mathrm{C}\right.$ ) and had a faint, blanching maculopapular rash over her torso, which became more confluent over the following days (Figure A). A full blood examination showed mild lymphopenia (lymphocyte count, $0.6 \times 10^{\circ} / \mathrm{L}$ [reference interval (RI), $\left.1.0-4.0 \times 10^{9} / \mathrm{L}\right]$ ). Mild hepatitis was also noted with an elevated alanine aminotransferase level of $120 \mathrm{U} / \mathrm{L}(\mathrm{Rl},<33 \mathrm{U} / \mathrm{L})$. Three sets of thick and thin blood films were sent to the laboratory, as were three sets of blood cultures along with stool and urine samples for culture and microscopy. Serological tests were performed for dengue fever virus and NSI antigen as well as for chikungunya virus. A throat swab was sent for respiratory virus multiplex polymerase chain reaction (PCR) testing, and droplet contact precautions were instituted in managing the patient.

Two days after she presented to hospital, the patient developed conjunctivitis, associated with progression of the rash to involve her face. Examination of the buccal mucosa at this stage revealed clusters of white granules (Figure B) consistent with classic Koplik spots, allowing a clinical diagnosis of measles to be made. A throat swab for measles virus PCR testing was subsequently taken, and the diagnosis was confirmed. The genotype of the measles virus was consistent with that of other index cases originating from Bali.

In accordance with the guidelines of the Victorian Department of Health $(\mathrm{DOH}){ }^{1}$ contact tracing was performed on 128 patients and their family members potentially exposed in the emergency department before the patient was isolated. Under $\mathrm{DOH}$ protocols, 18 contacts received prophylactic vaccination with the measles, mumps and rubella (MMR) live attenuated vaccine and four received normal human immunoglobulin $0.5 \mathrm{~mL} / \mathrm{kg}$ (maximum, $15 \mathrm{~mL}$ ). To date, the DOH has not identified any secondary cases.

Two days later, the patient's condition had improved clinically and the Koplik spots on the buccal mucosa had resolved (Figure C). She remained in hospital for 5 days for monitoring of probable measles hepatitis and to prevent further community exposure. Stool culture subsequently grew Campylobacter jejuni; this did not require antibiotic therapy and explained the initial clinical presentation with a diarrhoeal illness that preceded the onset of the typical measles rash and conjunctivitis.
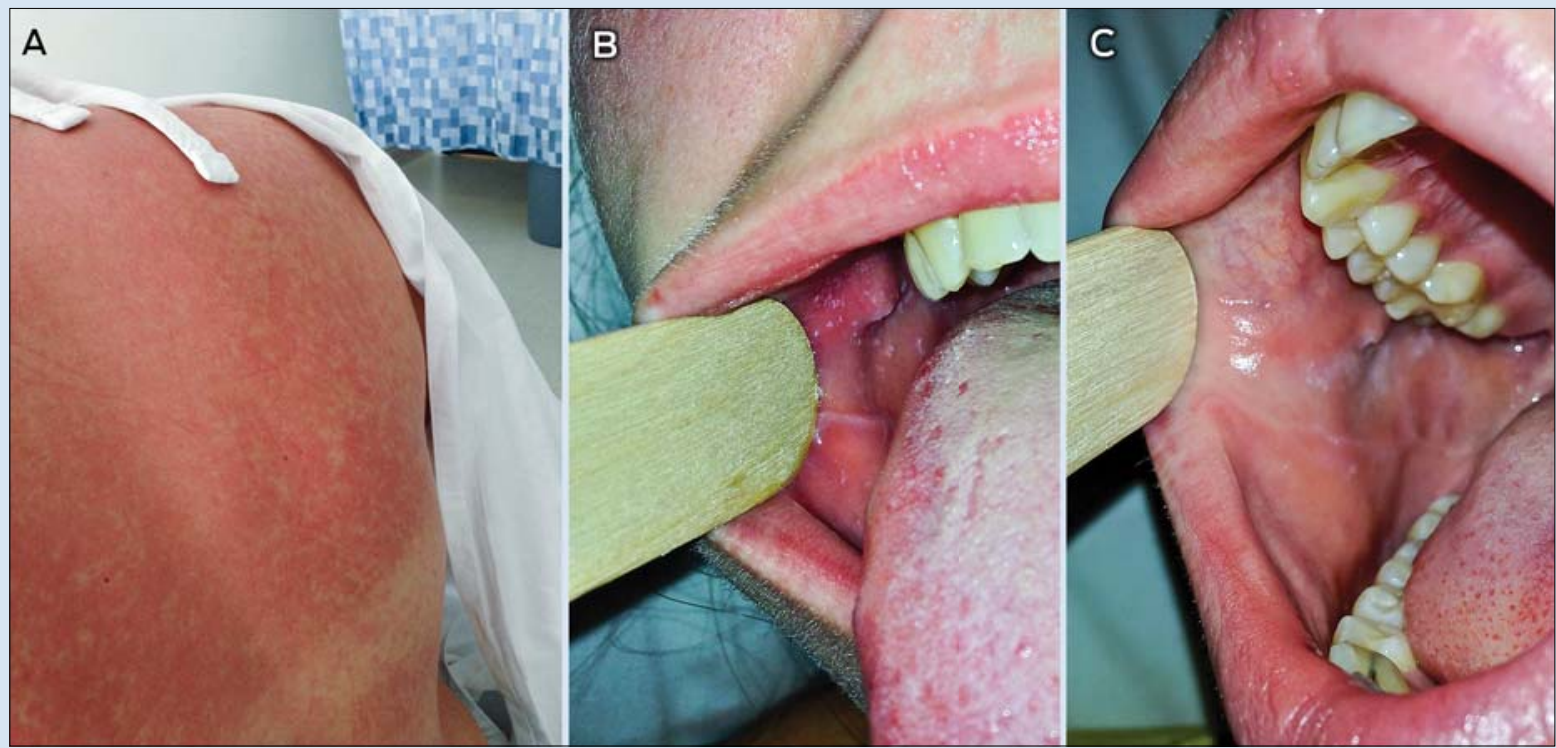

easles is a highly contagious RNA virus transmitted via respiratory secretions and aerosol. The incubation period is typically of 10-14 days duration, and it is followed by a prodrome of $2-4$ days with the development of fever, cough, conjunctivitis and coryza. At this stage, Koplik spots may be visible on the buccal mucosa and may persist for a few days before coalescing or sloughing. Koplik spots are a pathognomonic sign of measles and were first described in 1896 by paediatrician
Henry Koplik. ${ }^{2}$ They have been described as "grains of salt on a red background". ${ }^{3}$ The exanthematous phase that follows is characterised by a maculopapular rash, usually beginning on the face before becoming generalised. A number of complications can occur, the most serious of which include measles encephalitis or the much rarer delayed-onset subacute sclerosing panencephalitis.

In our case, concurrent Campylobacter gastroenteritis was thought to be the reason for the unusual initial 


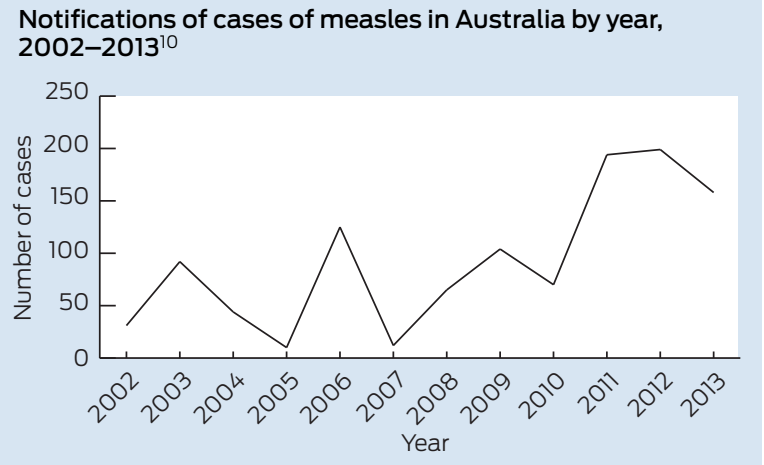

presentation with diarrhoeal symptoms, and why measles was not initially considered. Consequently, there was substantial exposure of staff and patients in the emergency department to this patient's infection. The development of conjunctivitis, progression of the rash, and the presence of Koplik spots prompted the initiation of contact tracing based on a clinical diagnosis, and before the confirmatory PCR result. This proved to be crucial in being able to administer the MMR vaccine to susceptible contacts in the 72-hour postexposure window.

Endemic measles has been eliminated from Australia for some time. ${ }^{4}$ However, sporadic cases continue to occur in non-immune travellers, their immediate contacts, and others in subsequent chains of transmission.

Measles vaccination was licensed in Australia in 1968, and people born before 1966 may generally be considered immune because they are likely to have been exposed to circulating wild-type virus. ${ }^{5}$ In Australia, about $92 \%$ of children have received two doses of MMR by 60 months of age, but vaccination coverage is lower in certain regions. ${ }^{6}$ There have been recent measles outbreaks in several European countries because of a decline in vaccination rates. ${ }^{7}$ As measles is one of the most highly transmissible infectious diseases known, with an estimated basic reproductive number (the average number of cases generated by one case in a susceptible population) of 12 to $40,{ }^{8}$ very high rates of vaccine coverage are required to prevent local outbreaks. ${ }^{9}$

Measles notifications in Australia have been trending upwards in the past few years (Box). Of the 37 Victorian

\section{Lessons from practice}

- Measles is an important and often missed part of the pretravel consultation; it should be considered a routine aspect of travel vaccination decision making.

- Consider measles as a differential diagnosis in febrile returned travellers born after 1966, especially if vaccination records are incomplete and the incubation and clinical presentation are consistent.

- Early isolation with airborne precautions is recommended in all potentially measles-susceptible patients who present with fever and rash. It can help to minimise the cost of subsequent contact tracing and measles prophylaxis. cases notified in 2013, 16 were imported cases and 20 were secondary or tertiary cases linked to an overseasacquired case. None of the Victorian patients with notified cases were known to be fully vaccinated. The most common countries of acquisition were Indonesia and Thailand (Victorian DOH, unpublished data, April 2014). From January to May 2014, 209 cases have been notified in Australia, with all states and territories apart from Tasmania reporting cases. This already exceeds the highest number of cases reported per year in Australia since 1999.

As was the case in our patient, ensuring that two documented doses of MMR vaccine are administered before travel is an often forgotten part of the pretravel consultation. A verbal recollection of vaccination or prior infection is often inaccurate. An alternative option is serological testing for the presence of measles IgG.

If diagnosis and appropriate infection control measures $^{11}$ are delayed, follow-up of contacts can be resource intensive. In Victoria, the DOH traces and manages community contacts. However, health care-associated exposures remain the responsibility of the individual institution. In cases such as ours, the cost and resources involved can be substantial.

Acknowledgements: We acknowledge the Communicable Disease Epidemiology and Surveillance Section, Victorian Department of Health for providing data.

Competing interests: No relevant disclosures.

1 Australian Government Department of Health and Ageing. Measles: national guidelines for public health units. Canberra: DoHA, 2009. http:// www.health.gov.au/internet/main/publishing.nsf/Content/cdna-songmeasles.htm (accessed Aug 2014).

2 Koplik $\mathrm{H}$. The diagnosis of the invasion of measles from a study of the exanthema as it appears on the buccal mucous membrane. Arch Pediatr 1896; 13: 918-922.

3 Bernstein DI, Schiff GM. Measles. In: Gorbach SL, Bartlett JC, Blacklow NR, editors. Infectious Diseases. Philadelphia: Saunders, 1998: 1296.

4 Heywood AE, Gidding HF, Riddell MA, et al. Elimination of endemic measles transmission in Australia. Bull World Health Organ 2009; 87: 64-71.

5 Australian Technical Advisory Group on Immunisation. The Australian immunisation handbook. 10th ed. Canberra: Australian Government Department of Health and Ageing, 2013. http://www.immunise.health. gov.au/internet/immunise/publishing.nsf/Content/Handbookl0-home (accessed Aug 2014).

6 National Centre for Immunisation Research and surveillance. Coverage information: The Australian Childhood Immunisation Register. http://www. ncirs.edu.au/immunisation/coverage/index.php (accessed Apr 2014).

7 Cottrell S, Roberts RJ. Measles outbreak in Europe. BMJ 2011; 342: d3724.

8 Bonacic Marinovic AA, Swaan C, Wichmann O, et al. Effectiveness and timing of vaccination during school measles outbreak. Emerg Infect Dis 2012; 18: 1405-1413.

9 Gay NJ. The theory of measles elimination: implications for the design of elimination strategies. J Infect Dis 2004; 189 Suppl 1: S27-S35.

10 Australian Government Department of Health. National Notifiable Diseases Surveillance System. http://www9.health.gov.au/cda/source/cda-index. cfm (accessed Feb 2014).

11 The Blue Book. Guidelines for the control of infectious diseases. Melbourne: Victorian Government Department of Human Services Communicable Diseases Section, 2005. http://docs.health.vic.gov.au/docs/doc/FE2665DB 66894C46CA2578B0001BE87E/\$FILE/bluebook.pdf (accessed Feb 2014). 口 